\title{
Transarterielle periartikuläre Embolisation (TAPE): klinischer Einsatz und erste Erfahrungen in Deutschland
}

\section{Transarterial Periarticular Embolization (TAPE): Indications and Initial Experience in Germany}

Autoren

Marcus Katoh ${ }^{1}$, Peter Schott ${ }^{1}$, Patrick Freyhardt ${ }^{1}$, Ludger Feyen ${ }^{1}$, Henrike-Renate Ziegler ${ }^{1}$, Clayton Kraft ${ }^{2}$

Institute

1 Department of Diagnostic and Interventional Radiology, HELIOS-Hospital Krefeld, Germany

2 Department of Orthopaedic Surgery, Hand and Trauma Unit, HELIOS-Hospital Krefeld, Germany

Key words

Transarterial periarticular embolization (TAPE), chronic joint pain, osteoarthritis, tendinopathy

eingereicht 25.05 .2020

akzeptiert 19.06.2020

Bibliografie

Fortschr Röntgenstr 2020; 192: 1046-1052

Online-Publikation: 3.9.2020

DOI $10.1055 / a-1212-6149$

ISSN $1438-9029$

(C) 2020. Thieme. All rights reserved.

Georg Thieme Verlag KG, Rüdigerstraße 14,

70469 Stuttgart, Germany

Korrespondenzadresse

Prof. Marcus Katoh

Diagnostische und Interventionelle Radiologie, Helios-

Klinikum Krefeld, Lutherplatz 40, 47805 Krefeld, Germany

Tel.: ++49/2151/322561

marcus.katoh@helios-gesundheit.de

\section{ZUSAMMENFASSUNG}

Ziel Anhand von Fallbeispielen sollen die Anwendungsmöglichkeiten, Technik und initialen Ergebnisse der transarteriellen periartikulären Embolisation (TAPE) dargestellt werden.

Material und Methoden Die TAPE wurde bei 3 Patienten mit chronischen Schmerzen an unterschiedlichen Gelenken angewendet. Im ersten Fall lag eine aktivierte Arthrose des Schultergelenks, im zweiten Fall eine Epicondylitis humeri ulnaris („Golfer-Ellenbogen“) und im dritten Fall ein Patellaspitzensyndrom (,Jumpers Knee“) vor.

Ergebnisse Bei allen 3 Patienten konnte die TAPE technisch erfolgreich durchgeführt werden. Dabei wurden entweder durch einen transradialen oder transfemoralen Zugang die Endäste der entsprechenden Gelenkarterien superselektiv mittels Mikrokatheter sondiert und mit in Kontrastmittel auf- gelöstem Imipenem/Cilastatin embolisiert. Postinterventionell kam es bei der Behandlung des Kniegelenks zu einer Hautrötung, die sich innerhalb einer Woche wieder zurückbildete. Weitere Komplikationen traten nicht auf. Bei allen Patienten zeigte sich bereits am ersten postoperativen Tag eine Linderung der Beschwerden, die anhand von klinischen Tests und der subjektiven Einschätzung der Schmerzen auf einer visuellen Analogskala dokumentiert werden konnte.

Schlussfolgerung Die initialen Erfahrungen, die in unserer Klinik mit der TAPE gemacht werden konnten, sind ermutigend und zeigen, dass das Verfahren das Potenzial zur Erweiterung der gering invasiven Therapieoptionen bei Patienten mit therapierefraktären chronischen Gelenkschmerzen und Enthesiopathien hat.

\section{Kernaussagen}

- Die TAPE ist eine noch junge Therapie zur Behandlung von Gelenkschmerzen und Enthesiopathien unterschiedlicher Genese.

- Die TAPE ist eine technisch anspruchsvolle endovaskuläre Intervention, die eine hohe Expertise voraussetzt.

- Die TAPE hat das Potenzial, das Behandlungsspektrum von Patienten mit chronischen Gelenkschmerzen zu erweitern.

\section{ABSTRACT}

Purpose The purpose of this article is to demonstrate the potential indications, procedural technique and initial results of the transarterial periarticular embolization (TAPE).

Material and Methods TAPE was performed in three patients with chronic pain in different joints. In the first case the patient suffered from osteoarthritis of the shoulder, in the second case from epicondylitis humeri ulnaris ("golferelbow") and in the third case from patellar tendinitis ("jumpers-knee"). Clinical as well as pain assessment was performed pre and post-interventionally.

Results TAPE was performed with technical success in all three patients. For vessel access, either a transradial or transfemoral access was chosen. The joint supplying vessels were catheterized superselectively with microcatheters and embolized with Imipenem/Cilastatin diluted in contrast medium. After embolization of the knee the patient demonstrated skin redness, which disappeared within one week. No further 
complications were noted. All patients reported significant pain relief within the first day after intervention.

Conclusion TAPE is a novel therapy for the treatment of persistent, chronic joint pain and tendinopathies, supported by publications from institutes outside of Europe. The initial experiences made in our institute are encouraging and suggest that TAPE may have the potential as an adjunct therapy option for patients with therapy-resistant chronic joint and tendinopathy-pain.

\section{Key Points}

- TAPE is a novel therapy for treatment of degenerative joint pain and tendinopathies
- TAPE is a technically challenging endovascular procedure and requires high interventional expertise

- TAPE may have the potential to develop to a minimallyinvasive therapy option for patients with chronic joint pain

\section{Citation Format}

- Katoh M, Schott P, Freyhardt P et al. Transarterial Periarticular Embolization (TAPE): Indications and Initial Experience in Germany. Fortschr Röntgenstr 2020; 192: 10461052

\section{Einleitung}

Die endovaskuläre Embolisation von Gefäßen an Gelenken ist ein Therapieansatz, der zunächst als Behandlungsoption für Patienten mit Hämarthros beschrieben wurde [1]. In diesen Studien konnte gezeigt werden, dass Patienten mit einem iatrogenen Hämarthros, z. B. nach Arthroskopie bzw. Arthroplastik, mit einer technischen Erfolgsrate von bis zu $100 \%$ und einer klinischen Erfolgsrate von bis zu 93 \% behandelt werden können. 2013 erschien die erste Arbeit, die zeigte, dass Gelenkschmerzen, die durch eine Tendinopathie oder Enthesiopathie ausgelöst werden, mittels einer endovaskulären Embolisation behandelt werden können [2]. 2015 folgte eine Arbeit, die den Einsatz der endovaskulären Embolisation zur Behandlung von Patienten mit Schmerzen im Rahmen einer Osteoarthritis beschrieb [3]. In dieser monozentrischen Studie wurde erstmalig gezeigt, dass nach der Embolisation die Schmerzlinderung bis zu einem Jahr anhalten kann. Diese Ergebnisse konnten in einer Wiederholungsstudie an einem größerem Patientenkollektiv von 72 Patienten bestätigt werden [4]. Es folgten weitere Studien, die den schmerzlindernden Effekt der Embolisation auch am Schulter- bzw. Ellenbogengelenk demonstrierten [5-7].

Ziel dieser Arbeit ist es, die Einsatzmöglichkeiten der transarteriellen periartikulären Embolisation (TAPE) zur Behandlung von chronischen Gelenkschmerzen bzw. Enthesiopathien, das technische Prozedere und initiale Ergebnisse anhand von 3 klinischen Patientenbeispielen in Deutschland zu illustrieren.

\section{Fallbeispiele}

\section{Fall 1 - Schulter}

Bei einem 82-jährigen Patienten lag eine traumatisch bedingte Versteifung des rechten Schultergelenks vor. Aufgrund der rechtsseitigen Bewegungseinschränkung habe der Patient die linke Schulter überdurchschnittlich beansprucht, die nun so schmerzhaft sei, dass er im Alltag stark einschränkt sei. Trotz der Einnahme von nichtsteroidalen Antirheumatika (NSAR) hätten die Schmerzen im Verlauf der letzten 5 Jahre immer weiter zugenommen.
Bei der Untersuchung zeigt sich eine schmerzbedingte Bewegungseinschränkung insbesondere bei der Anteversion $\left(90^{\circ}\right)$ und Abduktion (passiv $80^{\circ}$ /aktiv $40^{\circ}$ ). Die Röntgen-Aufnahme der Schulter zeigte osteophytäre Ausziehungen am Humeruskopf und eine Verschmälerung des Subakromialraums mit begleitendem Humeruskopf-Hochstand (Kellgren-Lawrence-Grad 3). Der Schmerz wurde auf einer visuellen Analogskala (VAS) von 110 tagsüber mit 7 und nachts mit 8 angegeben. Der American Shoulder and Elbow Surgeons (ASES) Score (der Score reicht von 0-100, wobei ein höherer Score geringere Schmerzen und eine bessere Funktionalität beschreibt) betrug 34,3.

In Zusammenschau aller Befunde wurde die Diagnose einer aktivierten Omarthrose gestellt.

\section{Fall 2 - Ellenbogen}

Ein 51-jähriger Patient stellte sich mit Schmerzen am Epicondylus humeri ulnaris vor, die durch eine beruflich und sportlich bedingte Überbelastung ausgelöst worden waren. Die Einnahme von NSAR und die Durchführung einer Physiotherapie hatten zu keiner Symptomlinderung geführt. 2 Jahre vorher sei aufgrund der Therapieresistenz schließlich, beim Verdacht auf ein N.-ulnaris-Rinnensyndrom, eine Neurolyse des Nervus ulnaris durchgeführt worden. Trotz der Operation seien die Beschwerden jedoch unverändert in Form eines Spannungsschmerzes im gesamten Gelenk und eines Druckschmerzes mit Punctum maximum über dem Epicondylis humeri ulnaris persistent.

Bei der Untersuchung zeigt sich ein schmerzhaftes, jedoch frei bewegliches Ellenbogengelenk rechts. Es fand sich eine vollkommen reizlose ehemalige Operationsnarbe. Hinweise für eine N.-ulnaris-Kompression zeigten sich nicht. Die Intensität des Schmerzes wurde auf der VAS mit 8 angegeben. Der Disabilities of the Arm, Shoulder and Hand (DASH) Score (der Score reicht von 0100, wobei ein höherer Score stärkere Symptome und Funktionseinschränkungen beschreibt) betrug 44,2.

Die MRT-Untersuchung des Ellenbogengelenks zeigte einen Reizerguss sowie eine leicht verdickte und kontrastmittelaffine Synovialis als Hinweis auf ein entzündliches Geschehen.

In Zusammenschau aller Befunde wurde die Diagnose einer chronischen Epicondylitis humeri ulnaris rechts („Golfer-Ellenbogen“) gestellt. 

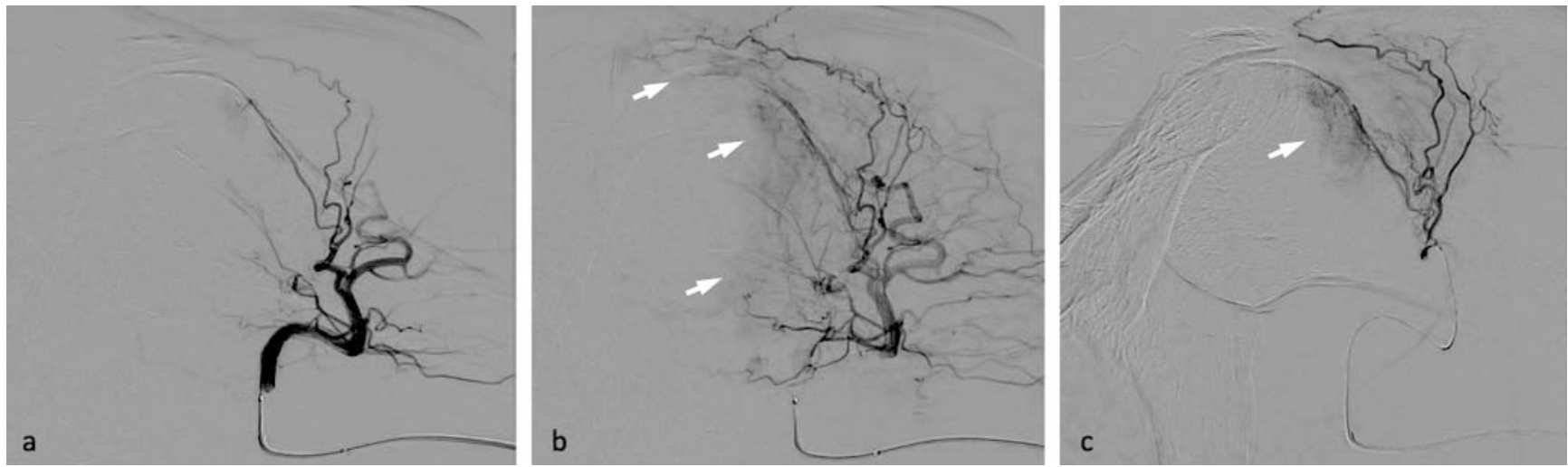

- Abb. 1 DSA des linken Schultergelenks bei einem Patienten mit aktivierter Arthrose nach Sondierung der A. circumflexa humeri posterior in einer sehr frühen a und einer etwas späteren b Kontrastmittelphase. In der späteren Kontrastmittelphase kommen deutliche „Kontrastmittelwolken“ zur Darstellung als Hinweis auf eine Hyperämie (Pfeile). Nach tiefer superselektiver Sondierung c des Gefäßes demarkiert sich deutlich ein hypervaskularisiertes Areal in Projektion auf den lateralen Anteil des Humeruskopfes (Pfeil).

- Fig. 1 DSA of the left shoulder joint in a patient with activated osteoarthritis after probing the posterior circumflex humeral artery in a very early a and somewhat later b contrast phase. In the later contrast phase, obvious contrast medium "clouds" appear as an indication of hyperemia (arrows). After deep superselective probing $\mathbf{c}$ of the vessel, a hypervascularized area is clearly demarcated in projection on the lateral part of the humeral head (arrow).

\section{Fall 3 - Knie}

Eine 23-jährige Patientin stellte sich mit Schmerzen im rechten Knie vor. Die Patientin habe seit ihrer Kindheit Tanzen als Leistungssport betrieben. Vor 5 Jahren seien erstmalig Schmerzen mit Punctum maximum am Unterpol der Patella aufgetreten. Diese seien in den letzten Monaten immer stärker geworden. Zuletzt seien diese so stark gewesen, dass sie nicht nur den Tanzsport aufgeben musste, sondern auch im alltäglichen Leben (wie z. B. beim Treppensteigen) erheblich eingeschränkt war.

Zunächst wurden bei der Patientin eine Medikation mit NSAR und Physiotherapie durchgeführt, die jedoch zu keiner signifikanten Linderung führten. Ferner wurden Injektionen mit einem kristalloiden Kortison peritendinös am distalen Patellapol durchgeführt. Bei ausbleibender Besserung wurde zuletzt eine operative Denervierung diskutiert.

Bei der Untersuchung zeigte sich ein frei bewegliches rechtes Kniegelenk. Es konnte ein starker Druckschmerz am Patella-Unterpol ausgelöst werden, der sich unter Lastaufnahme verstärkte. Eine MRT des Kniegelenks zeigte eine Hyperintensität der posterioren Anteile der Patellarsehne sowohl in der T1- als auch PDSequenz als Hinweis auf eine Degeneration und Ödem. Ein Knochenmarködem oder Gelenkerguss waren nicht erkenntlich. Arthrotische Veränderungen waren ebenfalls nicht vorhanden (Kellgren-Lawrence-Grad 0). Der Western Ontario and McMaster Universities Osteoarthritis Index (WOMAC) Score (der Score reicht von 0-96, wobei ein höherer Score stärkere Symptome und Funktionseinschränkungen beschreibt) wurde mit 68,2 ermittelt. Der Schmerz wurde auf der VAS mit 10 angegeben.

In Zusammenschau aller Befunde wurde die Diagnose einer chronischen Tendinitis der Patellarsehne im Rahmen eines Patellaspitzensyndroms (,Jumpers Knee“) gestellt.

\section{Therapie und Verlauf}

Bei allen 3 Patienten wurde im Rahmen eines individuellen Heilversuchs und nach entsprechender Aufklärung eine TAPE durchgeführt.

Bei dem Patienten im Fall 1 wurde zur Behandlung der Schulter ein radialer Zugang gewählt. Unter sterilen Kautelen erfolgten die Lokalanästhesie mit Prilocain (Xylonest $1 \%$ ) und die retrograde Punktion der A. radialis. In diese wurde eine 3,3F-Schleuse (pfm medica, Super Sheath) eingebracht und $2500 \mathrm{IE}$ unfraktioniertes Heparin verabreicht. Über die Schleuse wurde ein 3,3F-Selektivkatheter in JR1-Konfiguration (pfm medical, Mongoose) bis in die A. subclavia bzw. A. axiallaris vorgeführt. Es folgte die digitale Subtraktionsangiografie (DSA) nach manueller Kontrastmittelinjektion (GE, Accupaque 350) zur Darstellung der Gefäßanatomie. Die Ostien der abgehenden Gefäße wurden mit dem Selektivkatheter sondiert. In Koaxial-Technik erfolgte dann die superselektive Sondierung der Gefäßäste mit einem 1,7F-Mikrokatheter (Medtronic, Echelon 10) und einem 0,014“-Mikrodraht (Stryker, Syncro-14). Die DSA in der superselektiven Position zeigte deutliche „Kontrastmittelwolken" als Zeichen einer Hyperämie, die insbesondere über die A. circumflexa humeri anterior et posterior, den Ramus coracoideus und die A. circumflexa scapulae gespeist wurden ( $\triangleright$ Abb. 1). Während der Injektion gab der Patient ein Druckgefühl und einen Schmerz im Bereich der Beschwerdestelle an, die sich nach wenigen Sekunden wieder zurückbildeten. Sowohl die Lokalisationen der Kontrastmittelanreicherungen als auch die Art der induzierten Schmerzen korrelierten mit der von dem Patienten vorbeschriebenen Schmerzsymptomatik. Die Gefäße, die eine Hyperämie zeigten, bzw. dort, wo ein typischer Schmerzreiz ausgelöst werden konnte, wurden anschießend langsam mit Portionen von bis zu 1,5 ml eines Kontrastmittel (10 ml GE Accupaque 350)-Imipenem/ Cilastatin-Gemisches (Fresenius-KABI, Imipenem/Cilastatin $500 \mathrm{mg} / 500 \mathrm{mg}$ ) embolisiert, bis eine deutliche Flussverlangsamung oder Stase im entsprechenden Gefäß eintrat. Die Gesamt- 

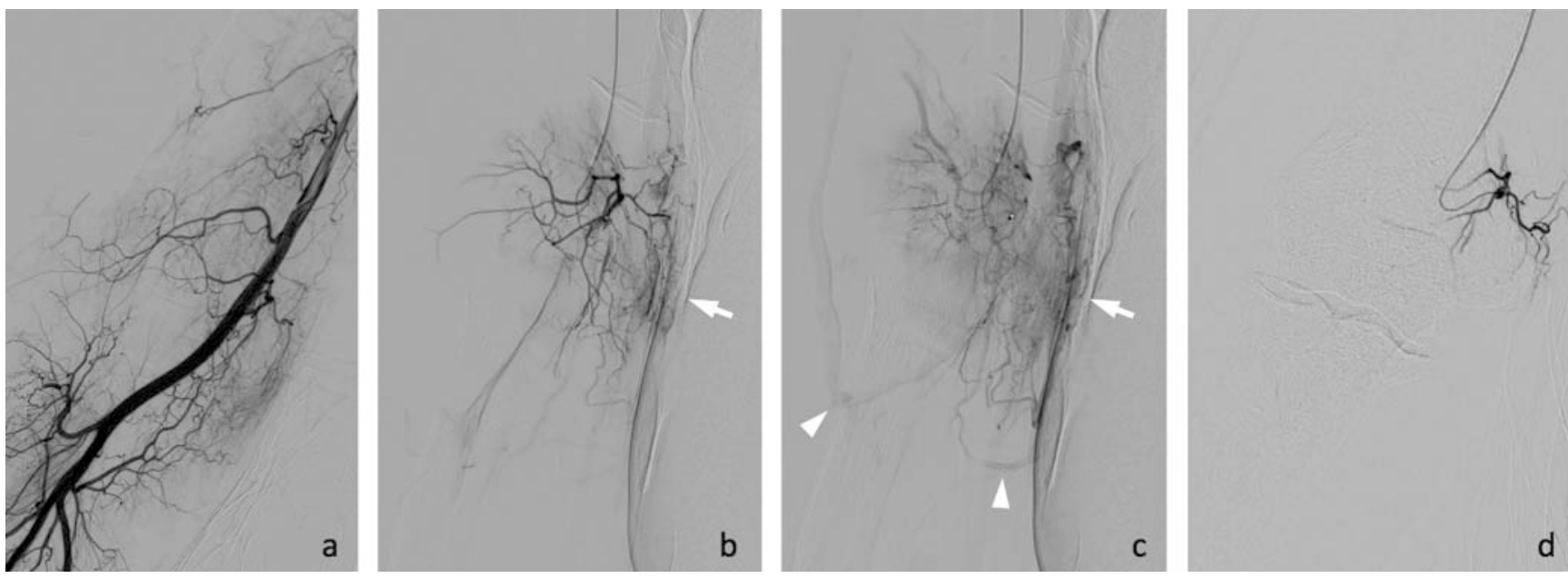

- Abb. 2 DSA des rechten Ellenbogengelenks bei einem Patienten mit Epicondylitis humeri ulnaris. Bereits in der Übersichtsangiografie a zeigt sich ein hypervaskularisiertes Areal in Projektion auf den Epicondylus humeri ulnaris. Dieses Areal (Pfeil) demarkiert sich deutlicher nach der superselektiven Sondierung u. a. der A. collateralis ulnaris superior b. In der späteren Kontrastmittelphase c zeigt sich eine charakteristische frühe Füllung der Venen (Pfeilspitzen). Nach Embolisation ist eine deutliche Flussverlangsamung zu erkennen; zudem stellen sich die hypervaskularisierten Areale nicht mehr dar $\mathbf{d}$.

- Fig. 2 DSA of the right elbow joint in a patient with epicondylitis of the ulnar humerus. The overview angiography a shows a strongly hypervascularized area in projection onto the epicondyle ulnar humerus. This area (arrow) is more clearly demarcated after the superselective probing of the superior ulnar collateral artery $\mathbf{b}$. In the later contrast phase $\mathbf{c}$ a characteristic early filling of the veins (arrowheads) is observed. There is a marked slowdown in flow after embolization; in addition, the hypervascularized areas are no longer visible $\mathbf{d}$.

menge des Embolisats betrug 5,5 ml (entspricht 275 mg Imipenem/275 mg Cilastatin). Nach Embolisation zeigte sich eine deutliche Reduktion, z. T. sogar eine komplette Rückbildung der Hyperämie. Nach Entfernung des Interventionsmaterials wurde die Punktionsstelle mit einem Kompressionsband (Terumo, TR Band) versorgt.

Der Patient konnte am ersten postinterventionellen Tag ohne Komplikationen entlassen werden. Bereits am Tag der Entlassung berichtete der Patient über eine deutliche Schmerzlinderung mit einem VAS-Score von 7 auf 4 bzw. Nachtschmerz von 8 auf 0 . Zudem konnte der Patient den Arm bereits durch die reduzierten Schmerzen besser bewegen; die maximale Anteversion betrug nun $180^{\circ}$ (vormals $90^{\circ}$ ) und die Abduktion $160^{\circ} / 120^{\circ}$ (vormals $80^{\circ} / 40^{\circ}$ ). Der ASES-Score verbesserte sich auf 63,3 (vorher 34,3).

Bei den Patienten im Fall 2 und Fall 3 wurde ähnlich wie im Fall 1 verfahren. Folgende Unterschiede ergaben sich jedoch: im Fall 2 wurde ein transfemoraler Zugang gewählt, um alle Gefäßabgänge $\operatorname{der} A$. brachialis, A. radialis und A. ulnaris sicher sondieren zu können. Über eine 4F-Schleuse wurde ein 4F-Selektivkatheter in Vertebralis-Konfiguration (Cordis, Tempo) bis in die A. subclavia rechts vorgeführt. In Koaxial-Technik wurden wie oben beschrieben die Gefäßabgänge mit einem 1,7F-Mikrokatheter (Medtronic, Echelon 10) und einem 0,014“-Mikrodraht (Stryker, Syncro-14) sondiert. In der Übersichtsangiografie zeigt sich eine stark hypervaskularisierte Gelenkkapsel, am stärksten jedoch in Projektion auf den Epicondylis humeri ulnaris. Dieses Areal wurde in erster Linie durch die A. collateralis ulnaris inferior und A. recurrens ulnaris versorgt ( $\boldsymbol{A} \mathbf{A b} \mathbf{b} . \mathbf{2}$ ). Es folgte die Embolisation der Gefäße mit dem o. g. Kontrastmittel-Imipenem/Cilastatin-Gemisch mit Portionen von bis zu 1,5 ml. Die Gesamtmenge des Embolisats betrug $4,5 \mathrm{ml}$ (entspricht $225 \mathrm{mg}$ Imipenem/225 mg Cilastatin).
Der Patient im Fall 2 konnte ebenfalls am ersten postinterventionellen Tag ohne Komplikationen entlassen werden und schilderte, dass das Druckgefühl und die Schmerzen des rechten Ellenbogengelenks deutlich nachgelassen hätten. Der Schmerz auf der VAS wurde mit nur noch 3 angegeben (vorher 8), der DASH-Score mit 15,0 (vorher 44,2).

Im Fall 3 wurde ebenfalls ein transfemoraler Zugang gewählt und eine 4F-Schleuse antegrad eingebracht. Nach Darstellung der Gefäßanatomie am Knie mittels einer 2-Ebenen-Angiografie erfolgte die superselektive Sondierung der Gefäßäste wie oben dargestellt. Bei der DSA zeigten sich stark hypervaskularisierte Areale in Projektion auf den Unterpol der Patella und den HoffaFettkörper, die in erster Linie über die A. inferior lateralis et medialis genus sowie die $A$. recurrens tibialis versorgt wurden $(\vee$ Abb. 3a-d). Auch hier wurde durch die Injektion des Kontrastmittels ein typischer Schmerz ausgelöst, der nach wenigen Sekunden wieder abklang. Es folgte die Embolisation der Gefäße mit dem o.g. Kontrastmittel-Imipenem/Cilastatin-Gemisch ( $\triangleright$ Abb.3e,f). Die Gesamtmenge des Embolisats betrug 6,0 ml (entspricht $300 \mathrm{mg}$ Imipenem/300 mg Cilastatin).

Bei der Patientin im Fall 3 zeigte sich unmittelbar postinterventionell eine Hautrötung ventral am Kniegelenk. Durch das Anlegen einer Kalt-Kompresse bildete sich diese teilweise zurück und war nach ca. einer Woche nicht mehr zu erkennen. Zu diesem Zeitpunkt gab die Patientin auch an, dass sie weitestgehend schmerzfrei, die Spannung bei der Kniebeugung nicht mehr zu spüren und dass auch das Treppensteigen wieder ohne Schmerzen möglich sei. Es wurde ein WOMAC-Score von 51 (vorher 68,2) ermittelt. Der Schmerz wurde auf der VAS mit 3 angegeben (vorher 10). 

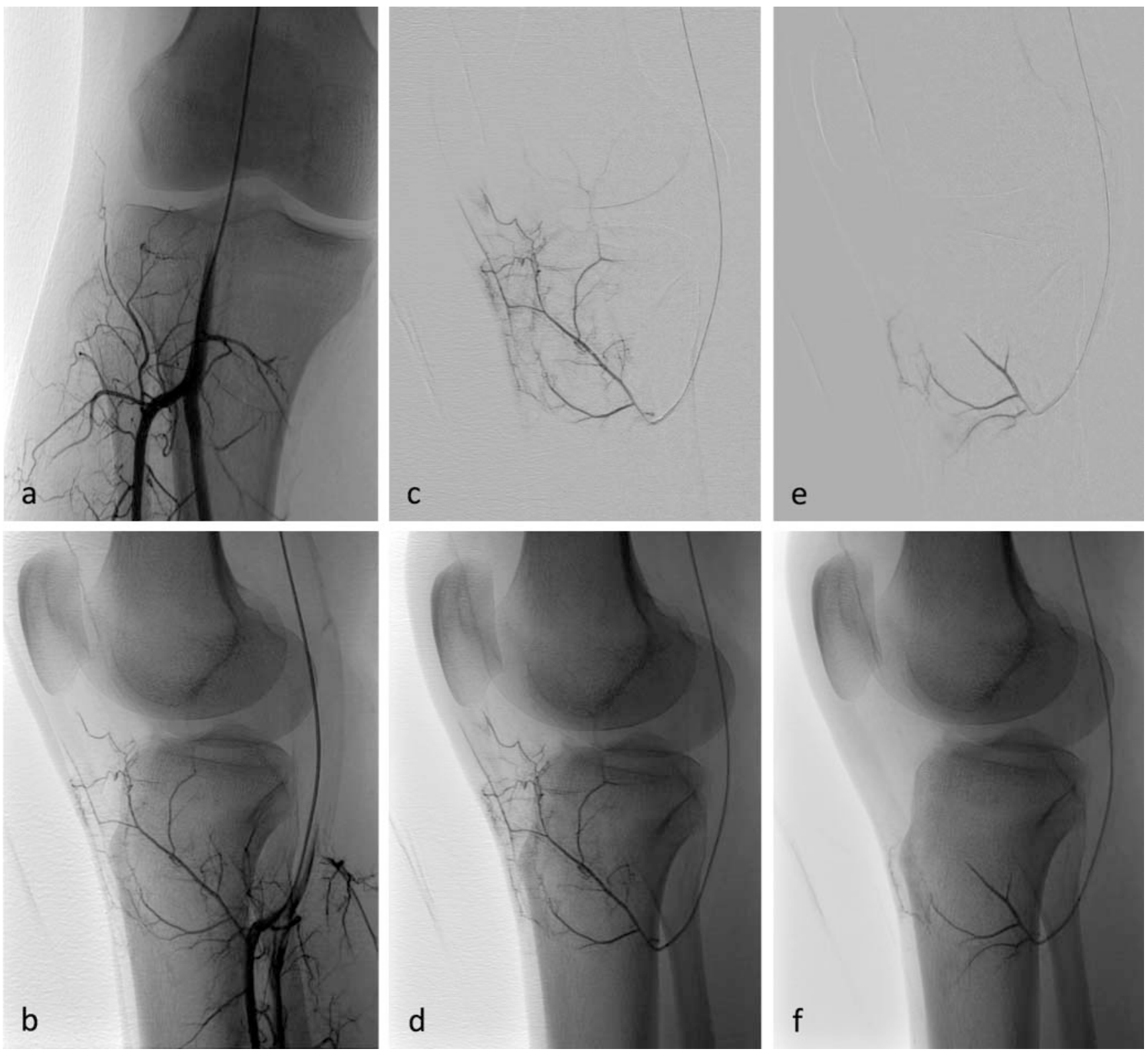

- Abb. 3 Übersichtsangiografie mit Lage der Katheterspitze im P3-Segment der A. poplitea (a a. p.; b seitlich). Die Angiografie im seitlichen Strahlengang nach superselektiver Sondierung der A. recurrens tibialis zeigt deutlich hypervaskularisierte Areale, die sich auf den Patella-Unterpol und den Hoffa-Fettkörper projizieren (c DSA; d nativ). Nach der Embolisation mit dem in Kontrastmittel aufgelösten Imipenem/Cilastatin, die bis zum Eintreten einer Stase durchgeführt wurde, kommen die hypervaskularisierten Areale nicht mehr zur Darstellung (e DSA; $\mathbf{f}$ nativ).

- Fig. 3 Overview angiography with position of the catheter tip in the P3 segment of the popliteal artery (a a. p.; b lateral). Angiography in the lateral beam path after superselective probing of the tibial recurrent artery clearly shows hypervascularized areas projecting onto the patella lower pole and the Hoffa fat body (c DSA; $\mathbf{d}$ native). The hypervascularized areas are no longer visible after embolization with imipenem/cilastatin dissolved in contrast medium, which was performed until stasis occurred, (e DSA; $\mathbf{f}$ native).

\section{Schlussfolgerung, Diskussion}

Bei allen 3 Patienten bestand ein chronisches Schmerzsyndrom eines Gelenks, das trotz einer intensiven orthopädisch begleiteten Behandlung über mehrere Jahre persistierte. Die Patienten wurden über die TAPE und der noch sehr limitierten Erfahrungen im Rahmen einer interdisziplinären Beratung ausführlich aufgeklärt. Sie willigten der Behandlung schließlich auf der Grundlage eines individuellen Heilversuchs ein.
Im Rahmen der Aufklärung wurden die Patienten auch darüber informiert, dass in den bisherigen Publikationen die TAPE entweder mit permanentem Embolisationsmaterial, wie z. B. Polyzenebeschichtete Hydrogel-Mikrosphären, oder mit temporärem Embolisationsmaterial, wie z. B. in Kontrastmittel aufgelöstes Imipenem/Cilastatin, durchgeführt wurde [4]. Die Anwendung des Antibiotikums Imipenem/Cilastatin als Embolisationsmaterial stellt trotz der Beschreibung in den genannten Arbeiten einen „Off-label-use“ dar. Dennoch wurde nach Abwägung der möglichen 
Komplikationen und Berücksichtigung der persönlichen Berichte der Autoren der bereits publizierten Studien sowohl von den behandelten Patienten als auch von den Autoren dieser Studie die Anwendung von Imipenem/Cilastatin favorisiert.

Eine der in den Studien beschriebenen Komplikationen sind Hautverfärbungen [4]. Auch wir konnten bei der Behandlung der Patientin mit dem Patellaspitzensyndrom postinterventionell eine deutliche Hautrötung feststellen, die sich jedoch nach Anlegen von Kühl-Kompressen relativ schnell zurückbildete und nach ca. einer Woche nicht mehr zu erkennen war. Bei der Anwendung von permanenten Partikeln wurde über deutlich längeranhaltende Hautverfärbungen berichtet [4, 7]. Im Fall eines Reflux könnten auch Non-Targeted-Embolisationen in die Akren nicht ausgeschlossen werden. Bei der TAPE im Bereich des Schultergelenks wären sogar Non-Targeted-Embolisationen in den Spinalkanal bzw. Rückenmark denkbar. Insbesondere unter dem Aspekt, schwerwiegende Komplikationen zu vermeiden, erscheint die Anwendung eines temporären Embolisats vorteilhaft. Als weiteres temporäres Embolisationsmaterial wären z. B. auch Gelatineschwamm- oder Stärke-Partikel denkbar; diese haben jedoch bislang in keiner der publizierten Studien Anwendung gefunden.

Überraschend ist die anhaltende schmerzlindernde Wirkung der Embolisation, auch wenn mit den temporären Embolisationsmaterialien nur ein vorrübergehender Verschluss der Gefäße erreicht wird. Es wird angenommen, dass der Schmerz durch pathologische, nicht myelinisierte sensorische Nerven vermittelt wird, die sich in der Umgebung von neu gebildeten Gefäßen bilden [8]. Die in entzündlich veränderten bzw. überbeanspruchten Strukturen wie der Gelenkkapsel, Synovialis, Periost und Sehnen stattfindende Neoangiogenese und Neoneurogenese wird durch lokoregionär freigesetzte proangiogenetische Faktoren wie z. B. VEGF/PDGF oder proneurogenetische Faktoren wie das beta-NGF induziert und unterhalten [9]. Diese Prozesse sind ferner verantwortlich für nachfolgende strukturelle Veränderungen bzw. Destruktionen von Knorpel und Knochen der Gelenke [10]. Es wird angenommen, dass durch eine Embolisation das Einschwemmen von inflammatorischen Zellen und proinflammatorische Zytokinen vermindert und damit die Stimulierbarkeit der Nerven herabgesetzt wird [3]. Ob diese Effekte allein durch die mechanische Okklusion der Gefäße bzw. durch die Erzeugung einer Ischämie bedingt sind, oder ob z. B. das kristalloide Antibiotikum Imipenem/Cilastatin möglichweise selbst eine antiinflammatorische oder analgetische Wirkung entfaltet, ist nicht bekannt.

Unerwartet war auch die schnell einsetzende Linderung der Beschwerden. Bei allen 3 dargestellten Fällen zeigte sich bereits am ersten postinterventionellen Tag eine deutliche Reduktion der Schmerzen, obwohl ihnen keine zusätzlichen Schmerzmittel verabreicht wurden. Bei dem Patienten mit den arthrotischen Schulterbeschwerden führte die Behandlung sogar zu einer deutlich verbesserten Beweglichkeit des Gelenks. Alle 3 Patienten berichteten zudem, dass sie auch nach 3 Monaten postinterventionell auf die Einnahme von Analgetika verzichten können.

Gelenkschmerz ist ein weitverbreitertes Leiden, dem meistens eine Arthrose, seltener auch aseptische oder septische Entzündungen zugrunde liegen [11]. Das therapeutische Spektrum beinhaltet die medikamentöse Einnahme von Analgetika, Physiotherapie oder Gelenkinjektionen [12]. Bei therapieresistenten arthrotischen Schmerzen ist häufig ein operativer prothetischer Gelenkersatz unausweichlich. Bei chronisch-entzündlichen Veränderungen von Sehnen oder Gelenkkapsel ist trotz der üblichen Therapieansätze ein längerer Krankheitsverlauf vorgezeichnet.

Die hier dargestellte TAPE stellt nach unseren ersten Erfahrungen eine vielversprechende neue Therapieoption zur Behandlung von Patienten mit chronischen Gelenkschmerzen dar. Die momentan noch spärliche Literaturdatenlage scheint dies zu unterstreichen. Das genaue Wirkungsprinzip der Embolisation ist derzeit noch unklar. Allerdings ist sehr gut denkbar, dass die TAPE die pathomorphologischen Veränderungen, die nach neuesten $\mathrm{Er}$ kenntnissen eine entscheidende Rolle bei degenerativ bedingten oder überlastungsbedingten Gelenkschmerzen spielen, nämlich die Neoangiogenese und Neoneurogenese, adressiert. Unter Würdigung der guten technischen Durchführbarkeit und der niedrigen Komplikationsrate erscheint es gerechtfertigt zu sein, den Ansatz weiter zu verfolgen. Dennoch gibt es wesentliche offene Fragen, die in zukünftigen Studien zu klären sein werden. Hierzu zählen die Identifikation des geeigneten Patienten, die Effektivität anderer Embolisationsmaterialien, das Komplikationsprofil in einem großen Patientengut und die Langzeitergebnisse der Therapie.

\section{KLINISCHE RELEVANZ}

- In Deutschland liegt die Prävalenz von Gelenkschmerzen bei $55,1 \%$ pro Jahr.

- Viele Patienten mit Arthrose leiden an therapieresistenten Gelenkschmerzen.

- Viele Patienten scheuen eine operative Gelenkersatztherapie.

- Insbesondere bei jüngeren Patienten ist aus orthopädischer Sicht die Gelenkersatztherapie problematisch.

- Die TAPE kann möglicherweise dazu beitragen, das Therapiespektrum von chronischen Gelenkschmerzen zu erweitern.

Interessenkonflikt

Die Autorinnen/Autoren geben an, dass kein Interessenkonflikt besteht.

Danksagung

We thank Dr. Yuji Okuno for his support in establishing the transarterial periarticular embolization in Germany

\section{Literatur}

[1] Waldenberger P, Chemelli A, Hennerbichler A et al. Transarterial embolization for the management of hemarthrosis of the knee. Eur J Radiol 2012; 81: 2737-2740

[2] Okuno Y, Matsumura N, Oguro S. Transcatheter arterial embolization using imipenem/cilastatin sodium for tendinopathy and enthesopathy refractory to nonsurgical management. J Vasc Interv Radiol 2013; 24: 787-792

[3] Okuno Y, Korchi AM, Shinjo T et al. Transcatheter arterial embolization as a treatment for medial knee pain in patients with mild to moderate osteoarthritis. Cardiovasc Intervent Radiol 2015; 38: 336-343 
[4] Okuno Y, Korchi AM, Shinjo T et al. Midterm Clinical Outcomes and MR Imaging Changes after Transcatheter Arterial Embolization as a Treatment for Mild to Moderate Radiographic Knee Osteoarthritis Resistant to Conservative Treatment. J Vasc Interv Radiol 2017; 28: 995-1002

[5] Okuno Y, Iwamoto W, Matsumura N et al. Clinical Outcomes of Transcatheter Arterial Embolization for Adhesive Capsulitis Resistant to Conservative Treatment. J Vasc Interv Radiol 2017; 28: 161-167.e1

[6] Iwamoto W, Okuno Y, Matsumura N et al. Transcatheter arterial embolization of abnormal vessels as a treatment for lateral epicondylitis refractory to conservative treatment: a pilot study with a 2-year follow-up. J Shoulder Elbow Surg 2017; 26: 1335-1341

[7] Hwang JH, Park SW, Kim KH et al. Early Results of Transcatheter Arterial Embolization for Relief of Chronic Shoulder or Elbow Pain Associated with Tendinopathy Refractory to Conservative Treatment. J Vasc Interv Radiol 2018; 29: 510-517
[8] Dye SF, Vaupel GL, Dye CC. Conscious Neurosensory Mapping of the Internal Structures of the Human Knee Without Intraarticular Anesthesia. The American Journal of Sports Medicine 2016; 26: 773-777

[9] Mapp PI, Walsh DA. Mechanisms and targets of angiogenesis and nerve growth in osteoarthritis. Nat Rev Rheumatol 2012; 8: 390-398

[10] Ayral X, Pickering EH, Woodworth TG et al. Synovitis: a potential predictive factor of structural progression of medial tibiofemoral knee osteoarthritis - results of a 1 year longitudinal arthroscopic study in 422 patients. Osteoarthritis and Cartilage 2005; 13: 361-367

[11] Fuchs ], Prütz F. Prevalence of joint pain in Germany, Robert Koch-Institut, Epidemiologie und Gesundheitsberichterstattung. Journal of Health Monitoring 2017; 2 (3). doi:10.17886/RKI-GBE-2017-056 Robert KochInstitut, Berlin

[12] Goldman DT, Piechowiak R, Nissman D et al. Current Concepts and Future Directions of Minimally Invasive Treatment for Knee Pain. Curr Rheumatol Rep 2018; Jul 23; 20 (9): 54 